\title{
Comparison of ankle range of motion and functional performance between practitioners of resistance exercise with free-weight vs. Machine
}

\begin{abstract}
The purpose of this investigation was to compare ankle functional performance and ankle range of motion (ROM) between practitioners of resistance exercise (RE) with free-weights versus machines. Twenty-five men participated in this study. They were separated into two groups: (a) Free-weights; and (b) Machines. All subjects practiced regularly RE $5.3 \pm 0.7$ $\mathrm{d} \cdot \mathrm{wk}-1$ and low aerobic training of $1.2 \pm 0.5 \mathrm{~d} \cdot \mathrm{wk}-1$ with a total time volume of $254.9 \pm 9.4$ min.wk-1. ROM measurements were taken in both ankles with a digital goniometer. Active ankle-dorsiflexion and plantar flexion range of motion were measured with subjects lying prone with an extended knee on a standard treatment table. The rising on the heel and the rising on toes were used to assess endurance of the ankle dorsiflexor and plantar flexor muscles, respectively. Ankle functional stability was assessed with the Single Leg Hop Test in both limbs. Ankle-dorsiflexion ROM showed a significant difference $(\Delta \%$ left $=21.1 \%$; $\Delta \%$ right $=25.8 \% ; \mathrm{P}<0.01$ ) between the Machines Group when compared to the Freeweights Group. Rising on the heel and rising on the toes showed no significant differences between the 2 groups (i.e., free-weights versus machines) $(\mathrm{P}>0.05)$. On the other hand, the Single Leg Hop Test $(\Delta \%$ left $=16.3 \% ; \Delta \%$ right $=15.4 \% ; \mathrm{P}<0.05)$ and number of jumps $(\Delta \%$ left $=27.9 \% ; \Delta \%$ right $=26.1 \% ; \mathrm{P}<0.05)$ recorded were lower in the Free-weights Group compared to the Machines Group. This study found a greater ankle-dorsiflexion ROM and performance during the Single Leg Hop Test in practitioners of RE with free-weights, showing a better control of sagittal plane movements.
\end{abstract}

Keywords: ankles, functional performance, range of motion, resistance training
Volume 4 Issue 3 - 2020

\author{
Alex Souto Maior,' Eduardo Lobo, ${ }^{2}$ Marcos \\ Braz, ${ }^{2}$ José Carlos de Campos Jr, ${ }^{2}$ Gustavo \\ Leporace $^{3}$ \\ 'Professor of the Master's and Doctorate Program in \\ Rehabilitation Science at UNISUAM (Augusto Motta University \\ Center), Brazil \\ ${ }^{2}$ UNISUAM (Augusto Motta University Center), Brazil \\ ${ }^{3}$ Coordinator of Biomechanics Research at Institute Brazil of \\ Technologies in Health, Brazil
}

Correspondence: Alex Souto Maior PhD,Augusto Motta University Center - UNISUAM, Postgraduate Program in Rehabilitation Sciences, Praça das Nações, 34 - Bonsucesso, ZIP Code 21041010 - Rio de Janeiro, RJ - Brasil, Email alex.bioengenharia@gmail.com

Received: June 06, 2020 | Published: July 212020

\section{Introduction}

Resistance exercises (RE) have been proposed in sports guidelines to improve physical conditioning and health ${ }^{1}$. The exercises represent a combination of dynamic actions and static effort. Specific devices are often used to evaluate muscle strength involving multiple variables that address training goals and individual needs (e.g., exercise order, rest interval between sets, exercise mode, training frequency, movement velocity, training volume, repetitions per set, number of sets, type of muscle action, and the load intensity). ${ }^{1-3}$ However, there are differences of opinion whether the use of a RE program that consist of free-weights or machines is better for building muscle mass, strength, and injury prevention. Free-weights utilize isotonic resistance that provides the same amount of resistance throughout the range of motion, thus free-weights allow for movement in multiple planes requiring balance., ${ }^{4,5}$ On the other hand, machines are a fixedform exercise that is limited to moving through fewer planes with a stable environment. ${ }^{1,4}$ But, interestingly, in regards to ankle range of motion (ROM) there are a limited number of studies that have assessed ankle-dorsiflexion and plantar flexion ROM when comparing RE with free-weights versus machines.

The ankle joint is formed by the dome of the talus fitting into a mortise formed by the tibia and the fibula where this joint produces movements of dorsiflexion and plantar flexion of the foot. Dorsiflexion is the movement at the ankle joint where the toes are brought closer to the shin, curling upwards, and decreasing the angle between the dorsum of the foot and the leg. ${ }^{6,7}$ On the other hand, plantar flexion describes the extension of the ankle so that the foot points down and away from the leg. ${ }^{6}$ A normal ankle moves from approximately $20^{\circ}$ dorsiflexion to $50^{\circ}$ plantar flexion in the sagittal plane and total range of motion in the frontal plane is approximately $35^{\circ}\left(23^{\circ}\right.$ inversion; $12^{\circ}$ eversion). ${ }^{6,89}$ Adequate ankle mobility is necessary for normal gait, as well as participation in many sporting and recreational activities.

Limited ankle-dorsiflexion ROM $\left(<11.5^{\circ}\right.$ with full knee extension and $<18.5^{\circ}$ with knee flexion of $90^{\circ}$ ) is often apparent to individuals with conventionally treated sprains. Without proper rehabilitation, the decrease in dorsiflexion ROM contributes to patellar tendinopathy, achilles tendinopathy, chronic ankle instability, metatarsal stress fractures, plantar fasciitis, anterior knee pain, and noncontact anterior cruciate ligament injuries. ${ }^{6,910,11}$ Ankle plantar flexors play a substantial role in the absorption of landing forces. During running the ankle plantar flexors support the body weight as well as propel the body forward in the late stance. ${ }^{12,13}$ Also, under conditions of the gastrocnemius, soleus and achilles tendon tightness, there is an increase in passive ankle stiffness as key factors that contribute to an increase in plantar loading $(12,22)$. To help avoid the increased loading, RE protocols seem to increase ankle-dorsiflexion and plantar flexion ROM. ${ }^{14,15}$

Current studies indicate that the ankle plantar flexors are important muscles to control for the mechanical work during the Free-Weights squat exercise while also using the exercise to strengthen the posterior-lateral muscles of the leg. However, if there is limited ankledorsiflexion during the squat exercise due to a low ankle plantar flexor net joint moments, the exercise is not very effective in increasing the ankle plantar flexor strength. The ROM of the ankle-dorsiflexion is a major factor affecting squat depth in both genders. ${ }^{16,17}$ The Free- 
Weights may be more beneficial than the Smith Machine squat for individuals who are looking to strengthen the plantar flexors. ${ }^{18}$ However, it is important to point out that little is known about the effect of the RE with Free- Weights versus Machines in ankle ROM. The absence of data supports the need for additional studies in this area. Hence, the purpose of this study was to compare ankle functional performance and ankle ROM between practitioners of RE with FreeWeights and Machines. We hypothesized that subjects trained with Free-Weights would show an increase in functional performance and ankle-dorsiflexion ROM.

\section{Methods}

\section{Subjects}

Twenty-five men were recruited and separated into two groups: Free-weights $(23.5 \pm 5.5$ years; $180.4 \pm 6.5 \mathrm{~cm} ; 82.1 \pm 6.8 \mathrm{~kg} ; 25.2 \pm 2.5$ $\left.\mathrm{kg} / \mathrm{m}^{2}, \mathrm{n}=12\right)$ and Machines $(25.3 \pm 4.5$ years, $179.1 \pm 5.3 \mathrm{~cm}, 80.6 \pm 6.2$ $\mathrm{Kg} ; 25.1 \pm 3.6 \mathrm{~kg} / \mathrm{m}^{2}, \mathrm{n}=13$ ). All subjects practiced regularly $\mathrm{RE}$ $5.3 \pm 0.7$ days week ${ }^{-1}$ and low aerobic training of $1.2 \pm 0.5$ day week $^{-1}$ with a total volume of $254.9 \pm 9.4$ minutes per week. Subjects with at least one year of RE experience were included to participate in the current study. Exclusion criteria included: (1) use of anabolic steroids, drugs, or medication with potential impact in physical performance (self-reported); (2) presence of musculoskeletal injury in the past 6 months and (3) previous hip, knee, and/or ankle surgery.

The Free-Weights Group performed a routine of RE that engaged the whole body with resistance bands, free-weights, and medicine balls. The Machine Group did their training routine of the whole body only with use of machines. All subjects completed the Physical Activity Readiness Questionnaire (PAR-Q). This study was approved by the Ethical Committee for Human Experiments of the Augusto Motta University Center, and was performed in accordance with ethical standards in sport and exercise science research (CAAE: 76189817.0.0000.5235). All subjects were informed of the experimental procedures and gave written informed consent prior to participation. No clinical problems occurred during the study.

\section{Ankle dorsiflexion and plantar flexion range of motion}

Measurements were taken in both ankles with a digital goniometer (Global Medical Devices; Maharashtra, India). Ankle-dorsiflexion and plantar flexion range of motion were measured with the subjects lying prone with an extended knee on a standard treatment table. This position was selected because both hip and knee joints are extended simultaneously, simulating the stance phase of gait just before heeloff. ${ }^{11}$ During assessments, all subjects wore shorts to provide adequate exposure to the ankle-foot-leg complex and were instructed to actively do a dorsiflexion and plantar flexion of the ankle joint (i.e., as far as comfortable without pain).

The rotational axis of the goniometer was placed just distal to the lateral malleolus, and the goniometer arms were aligned with the head of the fibula and parallel to the fifth metatarsal, respectively. The convention was followed of neutral position being $0^{\circ}$ and ankle motion being the number of degrees of angular movement from that position in either a dorsal or plantar direction. Measurements were recorded in degrees, and the mean score of 3 measurements was computed. This testing method has shown intra-rater reliability measures greater than 0.92 for the assessment of active ankle range of motion.

\section{Functional performance testing}

The rising on heel and rising on toes were used to assess endurance of the ankle dorsiflexor and plantar flexor muscles, respectively. ${ }^{6}$ Tests were performed on both ankles (right and left). For the rising on the heel test (plantar flexion), the subjects were instructed to perform the calf-raise movement continuously as many times as possible. For the rising on toes test (dorsiflexion), the subjects raised their toes (Figure 1). A pace of 60 repetitions. $\mathrm{min}^{-1}$, defined by the metronome, was used and the number of repetitions was the outcome measurement. During testing, the subjects were barefoot, heels on the ground, knees extended, without supported with the opposite knee at about $90^{\circ}$ flexion ${ }^{6}$. In cases of loss of balance, the subjects regained balance and the performance continued.

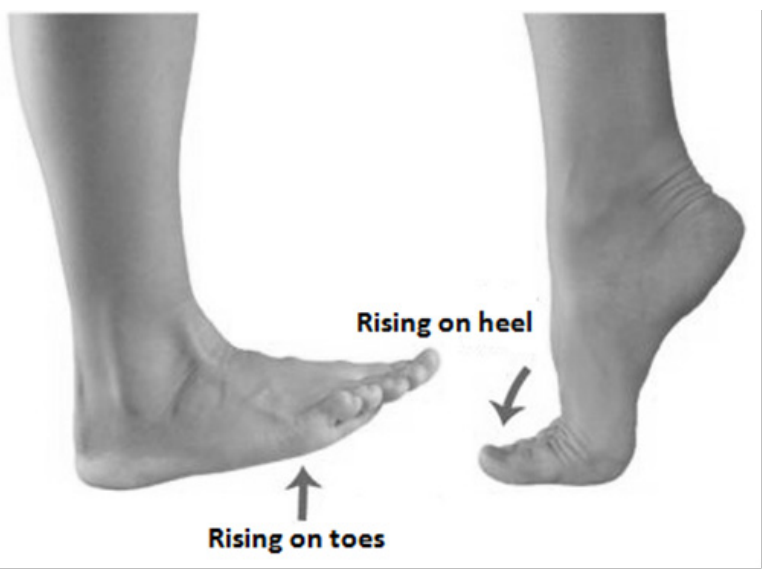

Figure I Ankle movement performance Testing.

Ankle functional stability was assessed with the single leg hop test performed bilaterally (right and left). Subjects, with footwear, positioned themselves single leg $30 \mathrm{~cm}$ behind of the first photocell beam (Brower Timing System, Salt Lake City, 174 UT, USA; accuracy of $0.01 \mathrm{sec})$. For the time record, subjects covered as fast as possible a 6-m distance that was timed by the second photocell beam. The test was repeated three times for both legs and a mean score of the 3 trials was then calculated. The subjects rested for $30 \mathrm{sec}$ between the trials. Verbal encouragement was always provided, and no subjects were excluded through injury during the experimental procedure. This test is was valid when it exhibits reliability that is higher than 0.90 .

\section{Statistical analysis}

All data are presented as mean \pm standard deviation. Statistical analysis was initially performed using the Shapiro-Wilk normality test and the homocedasticity test (Bartlett criterion). To test the reproducibility between the tests, the intraclass correlation coefficient (ICC) was used. Comparisons between groups (free weight vs. machine) and legs (right vs. left) were performed by the two-way ANOVA with Bonferroni post-hoc tests. The level of statistical significance was set at an alpha level of $\mathrm{P}<0.05$ using GraphPad Prism ${ }^{\circledR}$ software (Prism 6.0, San Diego, CA, USA).

\section{Results}

All analyzed data presented normal distribution. The two-way ANOVA yielded main effects for group $\left(F_{1,23}=13.01, p<.001\right)$, such that Bonferroni Post- hoc showed significant differences in the dorsiflexion to free weight group when compared to machine group 
$(\Delta \%$ left $=21.1 \% ; \Delta \%$ right $=25.8 \% ; p<.01)$ (Table 1$).$ In addition, dorsi/plantar flexion ratio also showed main effects for group $\left(F_{1,23}=\right.$ $10.58, p<.003)$ demonstrating that it was significantly lower in the Machine Group $(\Delta \%$ left $=35.1 \% ; \Delta \%$ right $=36.1 \% ; p<.01)$ (Table 1$)$. No significant difference $(p>.05)$ was found between the groups (freeweights vs. machines) to plantar flexion ROM.

Figure 2 summarizes the findings of the functional performance test for each group (Free-Weights vs. Machines). There were no significant differences $(p>0.05)$ between rising on the heel and rising

Table I Performance of ankle dorsiflexion and plantar flexion range of motion between practitioners' subjects of resistance exercise with free weight and machine $(n=25)$ on the toes between the Free-Weights and the Machines (Figure $2 \mathrm{~A}$ and $\mathrm{B})$. But, rising on the toes revealed main effects for group $\left(F_{1,46}=8.037 p<0.006\right)$. On the other hand, the Single Leg Hop Test and number of jumps showed main effects for group $\left(F_{1,23}=6.60, p<0.01\right.$; $F_{1,23}=8.40, p<0.008$, respectively), such that there were significantly lower values during the Single Leg Hop Test $(\Delta \%$ left $=16.3 \% ; \Delta \%$ right $=15.4 \% ; p<0.05)$ and number of jumps $(\Delta \%$ left $=27.9 \% ; \Delta \%$ right $=26.1 \% ; p<0.05)$ in the Free-Weights Group when compared to Machines Group (Figure 2).

\begin{tabular}{|c|c|c|c|c|c|}
\hline & & Free Weight & Machine & $\begin{array}{l}\text { Mean difference } \\
(95 \% \mathrm{Cl})\end{array}$ & $p<$ \\
\hline \multicolumn{6}{|l|}{ Dorsiflexion $\left({ }^{\circ}\right)$} \\
\hline & Left & $17.8 \pm 2.5$ & $14.7 \pm 2.1$ & $\begin{array}{l}3.31 \\
(0.89 \text { to } 5.73)\end{array}$ & $<.01$ \\
\hline & Right & $17.5 \pm 2.3$ & $13.9 \pm 1.6$ & $\begin{array}{l}3.82 \\
(1.41 \text { to } 6.24)\end{array}$ & $<.01$ \\
\hline \multicolumn{6}{|c|}{ Plantar Flexion $\left({ }^{\circ}\right)$} \\
\hline & Left & $36.0 \pm 4.2$ & $40.7 \pm 5.6$ & $\begin{array}{l}-4.18 \\
(-9.65 \text { to } 1.28)\end{array}$ & $>.05$ \\
\hline & Right & $36.1 \pm 4.5$ & $39.8 \pm 5.0$ & $\begin{array}{l}-2.84 \\
(-8.31 \text { to } 2.61)\end{array}$ & $>.05$ \\
\hline \multicolumn{6}{|c|}{$\begin{array}{l}\text { Dorsi/Plantar } \\
\text { flexion ratio (a.u.) }\end{array}$} \\
\hline & Left & $0.50 \pm 0.07$ & $0.37 \pm 0.08$ & $\begin{array}{l}0.12 \\
(0.03 \text { to } 0.22)\end{array}$ & $<.01$ \\
\hline & Right & $0.49 \pm 0.08$ & $0.36 \pm 0.08$ & $\begin{array}{l}0.12 \\
(0.03 \text { to } 0.21)\end{array}$ & $<.01$ \\
\hline
\end{tabular}

(A)

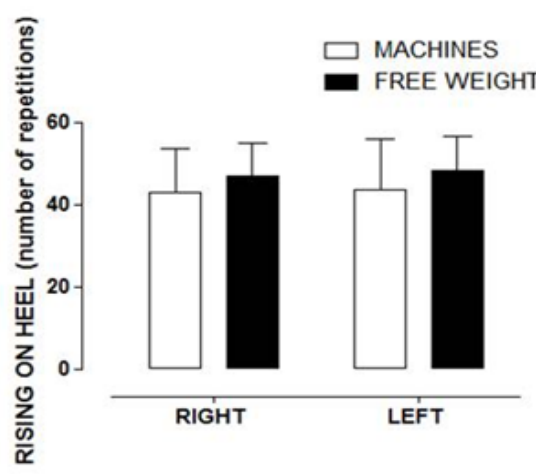

(C)

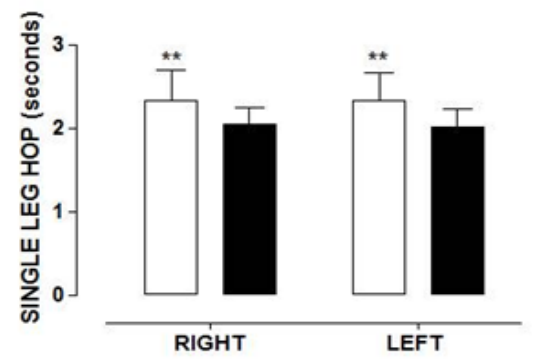

(B)

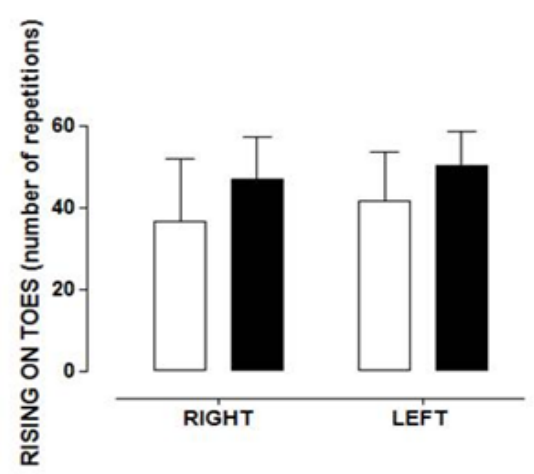

(D)

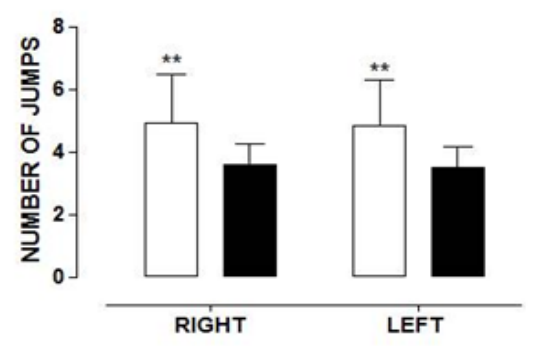

Figure 2 Values are expressed as mean \pm standard deviation. Rising on heel (A), rising on toes (B), single leg hops test (C) and number of jumps (D) during ankle specific test with free weight $(n=12)$ and machine $(n=13) . * *<.0 I-$ free-weights vs. machine. 


\section{Discussion}

The purpose of this study was to compare ankle functional performance and ankle range of motion (ROM) between practitioners of RE with Free-Weights versus Machines. The main findings are that the Free-Weights Group showed better mobility and stability of the ankles when compared to the Machines Group. The results of this study confirm that RE with free-weights may contribute to movement in multiple planes and requires more stabilization and balance., ${ }^{1,18}$ These factors seem to be very important for increasing range of motion, functional performance, and injury prevention of the ankles.

Ankle dorsiflexion motion is essential in multi-directional tasks to facilitate mobility, stability, and balance. On the other hand, a limited ankle-dorsiflexion ROM is associated with a greater dynamic knee valgus and medial knee displacement, as well as a reduced activation of the quadriceps and increased activation of the soleus. ${ }^{7,19}$ This limitation may be caused by factors such as heel-cord tightness and lack of arthrokinematic motion at the talocrural joint. ${ }^{20}$ However, scientific evidence has shown that decreased extensibility of the gastrocnemius/soleus complex and restricted posterior talar glide on the tibia contribute directly to a limited ankle-dorsiflexion ROM.,21 Furthermore, decreased strength of the ankle musculature decreases the ability to stabilize the lower extremity, resulting in a faulty alignment of the lower extremity (such as adduction and rotation of the hip and knee valgus). ${ }^{22}$

Our results indicate that the subjects trained with machines had limited dorsiflexion $(21.1 \%$ and $25.8 \%$ in the left and right ankles, respectively). A possible explanation might be that the exercises on the machines contribute with movements made only in a single plane of motion and that ballistic movements are nearly impossible to perform. ${ }^{1,4}$ Conversely, free-weights exercises are compound variable resistance that provides a load which changes to match the ability of the musculoskeletal lever system to produce force throughout the range of motion. ${ }^{21,23}$ Thus, the ankle is an important joint of the closed-chain movement during squatting activities, balance, mobility, and stability of the ankle. ${ }^{22}$ In relation to ankles injury prevention and rehabilitation, recent trends suggest the inclusion of more functional movement and more closed-kinetic-chain rather than open-kineticchain positioning in hopes of returning patients to functional activities and preventing future incidents of injury. ${ }^{24}$ Because the closedkinetic-chain exercise is a multi-joint movement with controlled cocontractions of synergistic and antagonistic muscles associated with minors anterior-posterior shear forces, there is less likelihood of an injury. ${ }^{19}$

The functional performance tests require agility to better represent functional movements necessary for athletic participation. Thus, the tests may be more difficult to perform with decreased stability and mobility of the ankles because the ankles are an important part of the closed-kinetic-chain movement. ${ }^{17,24}$ While the results did not show significant differences between the Free-Weights Group versus the Machines Group during rising on toes, the percent of total variation was $14.42 \%$ greater in the Free-Weights Group. No significant difference was also observed during rising on heel. In contrast, performance during the Single Leg Hop Test and the number of jumps showed minors values in the Free-Weights Group.

Although our results did not show a significant correlation between rising on toes and single leg hop test, it has been observed that limitations in the ankle-dorsiflexion ROM may contribute to a stiff landing with less flexion at the ankle and knee during the single leg drop. ${ }^{25}$ Consequently, limited ankle-dorsiflexion ROM is associated with persisting deficits in explosive leg power, agility, and proprioception. ${ }^{19}$ Furthermore, less ankle dorsiflexion ROM exhibits less knee flexion at initial contact, less maximum knee flexion during landing and may impair the knee and hip kinematics during different landing tasks. ${ }^{25,26}$ Due to the lack of comparative study between free weights vs. machines in the functional performance of the ankles, it was hypothesized that RE with machines could contributes to limited ankle-dorsiflexion because of a tight soleus, gastrocnemius, and/or capsular tissue that would result in a limited ankle functional performance during the Single Leg Hop Test.

A limitation of this type of investigation could be that the specific effects of resistance training on ankle-dorsiflexion ROM are unknown and further studies should examine the effect of first toe muscle strength. However, our sample was homogeneous although longitudinal studies are needed to define a cause-and-effect relationship.

This study found a greater ankle-dorsiflexion ROM and performance during the Single Leg Hop Test in practitioners of RE with free-weights, which showed a better control of sagittal plane movements and musculoskeletal injury prevention. This finding shows the efficiency of ankle-dorsiflexion ROM during functional performance of the ankles.

\section{Acknowledgments}

This study was supported by the Coordenação de Aperfeiçoamento de Pessoal de Nível Superior - Brasil (CAPES) - Finance Code 001.

\section{Declaration of conflicting interests}

The author(s) declared no potential conflicts of interest with respect to the research, authorship, and/or publication of this article.

\section{References}

1. American College of Sports Medicine. American College of Sports Medicine position stand. Progression models in resistance training for healthy adults. Med Sci Sports Exerc. 2009;41(3):687-708.

2. De Salles BF, Maior AS, Polito M, et al. Influence of rest interval lengths on hypotensive response after strength training sessions performed by older men. J Strength Cond Res. 2010;24(11):3049-3054.

3. Marocolo M, Marocolo IC, Cunha FSB, et al. Influence of percentage of $1 \mathrm{RM}$ strength test on repetition performance during resistance exercise of upper and lower limbs. Arch Med Deporte. 2016;33(6):387-392.

4. Stone M, Plisk S, Collins D. Training principles: evaluation of modes and methods of resistance training - a coaching perspective. Sports Biomech. 2002;1(1):79-103.

5. Welch N, Moran K, Antony J, et al. The effects of a free-weightbased resistance training intervention on pain, squat biomechanics and MRI-defined lumbar fat infiltration and functional cross-sectional area in those with chronic low back. BMJ Open Sport Exerc Med. 2015;1(1):e000050.

6. Lazarou L, Kofotolis N, Pafis G, et al. Effects of two proprioceptive training programs on ankle range of motion, pain, functional and balance performance in individuals with ankle sprain. $J$ Back Musculoskelet Rehabil. 2018;31(3):437-446.

7. Lima YP, Ferreira VMLM, Lima POP, et al. The association of ankle dorsiflexion and dynamic knee valgus: A systematic review and metaanalysis. Phys Ther Sport. 2018;29:61-69. 
8. Brockett CL, Chapman GJ. Biomechanics of the ankle. Orthop Trauma. 2016;30(3):232-238.

9. Kaufman KR, Brodine SK, Shaffer RA, et al. The effect of footstructure and range of motion on musculoskeletal overuse injuries. Am J Sports Med.1999;27(5):585-593

10. Fong CM, Blackburn JT, Norcross MF, et al. Ankle-dorsiflexion range of motion and landing biomechanics. J Athl Train. 2011;46(1):5-10.

11. Youdas JW, McLean TJ, Krause DA, et al. Changes in active ankle dorsiflexion range of motion after acute inversion ankle sprain. J Sport Rehabil. 2009;18(3):358-374.

12. Kawakami Y, Kanehisa H, Fukunaga T. The relationship between passive ankle plantar flexion joint torque and gastrocnemius muscleand achilles tendon stiffness: implications for flexibility. J Orthop Sports PhysTher. 2008;38(5):269-276.

13. Sakanaka TE, Gill J, Lakie MD, et al. Intrinsic ankle stiffness during standing increases with ankle torque and passive stretch ofthe Achilles tendon. PLoS One. 2018;13:e0193850.

14. Hall EA, Docherty CL, Simon J, et al. Strength-training protocols to improve deficits in participants with chronic ankle instability: a randomized controlled trial. J Athl Train. 2015;50(1):36-44.

15. Rajan P, Porter MM.Velocity during Strength and Power Training of the Ankle Plantar and Dorsiflexor Muscles in Older Patients Attending Day Hospital Rehabilitation. Rehabil Res Pract. 2015;2015:586843.

16. Chiu LZ, vonGaza GL, Jean LM. Net joint moments and muscle activation in barbell squats without and with restricted anterior leg rotation. J Sports Sci. 2017;35(1):35-43.

17. Kim SH, Kwon OY, Park KN, et al. Lower extremity strength and the range of motion in relation to squat depth. J Hum Kinet. 2015;45:5969.

18. Schwanbeck S, Chilibeck PD, Binsted G. A comparison of free weight squat to Smith machine squat using electromyography. J Strength Cond Res. 2009;23(9):2588-2591.
19. Dill KE, Begalle RL, Frank BS, et al. Altered knee and ankle kinematics during squatting in those with limited weight-bearing-lunge ankledorsiflexion range of motion. J Athl Train. 2014;49(6):723-732.

20. Krause DA, Cloud BA, Forster LA, et al. Measurement of Ankle Dorsiflexion: A Comparison of Active and Passive Techniques in Multiple Positions. J Sport Rehabil. 2011;20(3):333-344.

21. Yoon JY, Hwang YI, An DH, Oh JS. Changes in kinetic, kinematic, and temporal parameters of walking in people with limited ankle dorsiflexion: pre-post application of modified mobilization with movement using talus glide taping. J Manipulative Physiol Ther. 2014;37(5):320-325.

22. Schoenfeld BJ. Squatting kinematics and kinetics and their application to exercise performance. J Strength Cond Res, 2010;24(12): $3497-$ 3506 .

23. Baroni BM, Pompermayer MG, Cini A, et al. Full range of motion induces greater muscle damage than partial range of motion in elbow flexion exercise with free weights. J Strength Cond Res. 2017;31(8):2223-2230.

24. Webster KA, Gribble PA. Functional rehabilitation interventions for chronic ankle instability: a systematic review. J Sport Rehabil. 2010;19(1):98-114.

25. Dowling B, Mcpherson AL, Paci JM. Weightbearing ankle dorsiflexion range of motion and sagittal plane kinematics during single legdrop jump landing in healthy male athletes. J Sports Med Phys Fitness. 2018;58(6):867-874.

26. Leporace G, Tannure M, Zeitoune G, et al. Association between kneeto-hip flexion ratio during single-leg vertical landings, and strength and range of motion in professional soccer players. Sports Biomech. 2020;19(3):411-420. 